\title{
Size and Shape Dependent Catalytic Activation Energy of Different Nano Structures
}

\author{
Purvi A. Bhatt ${ }^{1}$, Arun Pratap ${ }^{2}$ and Prafulla K. Jha $^{3}$ \\ ${ }^{1,2}$ Condensed Matter Physics Laboratory, Applied Physics Department, Faculty of Technology \& \\ Engineering, The M. S. University of Baroda, Vadodara, India. \\ ${ }^{3}$ Department of Physics, Faculty of Science, The M. S. University of Baroda, \\ Vadodara, India.
}

Correspondence should be addressed to: Purvi A. Bhatt; purvi.physics.712@gmail.com

Received date: 12 May 2014; Accepted date: 22 July 2014; Published date: 28 January 2015

Academic Editor: Parthasarathi Bera

Copyright (C 2015. Purvi A. Bhatt, Arun Pratap and Prafulla K. Jha. Distributed under Creative Commons CC-BY 4.0

\begin{abstract}
In the present work, we report the effect of size and dimension on catalytic activation energy $\left(E_{C}\right)$ of some dioxide nano structured materials using a simple analytical model. We report the variation of catalytic activation energy with the size from $1 \mathrm{~nm}$ to $30 \mathrm{~nm}$ and $0-, 1-, 2-$ dimensions of $\mathrm{TiO}_{2}, \mathrm{CeO}_{2}$ and $\mathrm{SnO}_{2}$ nanoparticles. The catalytic activation energy decreases as the size of these nanoparticles decreases and is minimum for zero dimensional structure of all considered materials. We show that the nano sized $\mathrm{TiO}_{2}$ has the minimum value of catalytic activation energy indicates its being better catalyst than other nanostructures. The shape dependence of catalytic activation energy has also been discussed. The particles having tetrahedral shape exhibit minimum catalytic activation energy, thus they show better efficiency as catalysts.
\end{abstract}

Keywords: Catalysis, Activation energy, nanostructure, $\mathrm{TiO}_{2}, \mathrm{CeO}_{2}, \mathrm{SnO}_{2}$

\section{Introduction}

The size and shape dependent properties of nanomaterials have been extensively studied during the past few years. Once the size of the matter approaches to atomic scale, it exhibits fascinating electrical, optical, magnetic, vibrational, thermo dynamical and catalytic properties than its bulk counterpart resulting from salient features like large surface-to-volume ratio and quantum effect (Alivisatos et al., 1998, Alivisatos, 1996,
Gupta et al., 2009, Gupta et al., 2008, Gupta and Jha, 2009, Mankad et al., 2011, Talati and Jha, 2005, Gupta et al., 2010, Jha et al., 2006). Researchers have shown that the size and shape of a particle are key factors to influence the various phenomena including melting, interface energy, surface diffusion, catalysis, etc (Bhatt et al., 2012, Mishra et al., 2012, Gleiter, 2000, Zhang et al., 2000, Jiang and Lu, 2008, Jiang et al., 2004, Zhou et al., 2010, Gupta et al., 2008). 
In recent years, enormous efforts have been devoted to study the size and shape dependence of nanoparticles to understand the catalytic and electro catalytic performances (Narayanan and El-Sayed, 2004, Burda et al., 2005, Li et al., 2000). Nanoparticles of different materials act as efficient catalysts for oxidation of hydrocarbons, C-C coupling, hydrogenationdehydrogenation, redox and other chemical transformations (Zhou et al., 2010, Burda et al., 2005, Somorjai et al., 2006, Ertl et al., 1997, Bell, 2003, Chen and Goodman, 2006, Crooks et al., 2001, Lewis, 1993, Astruc et al., 2005, Roucoux et al., 2002, Schmid, 2004, Wasylenko and Frei, 2007, Kung et al., 2007). Past studies show that the titanium dioxide $\left(\mathrm{TiO}_{2}\right)$ is a promising candidate for the decomposition of wide variety of organic and inorganic moieties in both liquid and gas phases (Mankad et al., 2011, Gupta et al., 2010, Jha, 2013, Desai et al., 2011). The nano scale cerium dioxide $\left(\mathrm{CeO}_{2}\right)$ has also advantages as a support for catalysis since it can enhance the reactivity for the oxidation reaction and $\mathrm{CO}$ oxidation when supported to gold nanoparticles (Guo et al., 2010, Carrettin, 2004). Although nanoparticles of tin dioxide $\left(\mathrm{SnO}_{2}\right)$ are of less interest particularly for catalytic applications, Sharghi et al., (2006) have found that the $\mathrm{SnO}_{2}$ nanoparticles are highly reactive in the Knoevenagel condensation process.

Catalytic activation energy $\left(E_{C}\right)$ is one of the important kinetic parameters, which measures the chemical activity of the catalyst to catalyze different reactions (Narayanan and El-Sayed, 2004). In the present paper, we have calculated and compared the catalytic activation energy $\left(E_{C}\right)$ of three different nanostructures i.e., titanium dioxide $\left(\mathrm{TiO}_{2}\right)$, cerium dioxide $\left(\mathrm{CeO}_{2}\right)$ and tin dioxide $\left(\mathrm{SnO}_{2}\right)$. The size and shape dependent catalytic activation energy of $\mathrm{TiO}_{2}, \mathrm{CeO}_{2}$ and $\mathrm{SnO}_{2}$ nanostructures has been calculated using a simple model based on analytical approach. Further, to analyze the dimension dependency on the catalytic activity of these nanostructures, we have also calculated the activation energy for- 0-,
1- and 2- dimensional $\mathrm{TiO}_{2}, \mathrm{CeO}_{2}$ and $\mathrm{SnO}_{2}$ nanostructures that correspond to spherical nanoparticles, nanowires and thin films respectively. We observe that the different shapes have considerable effect on the catalytic activation energy. The paper is divided into four main sections. In section 2 , brief description of theoretical model is presented. Section 3 describes the results and discussion followed by a summary of the present work in section 4 .

\section{Methodology and Computation}

The catalytic activation energy in the present study is determined using a theoretical model based on an analytical approach which includes the shape factor (Lu and Meng, 2010). The size and temperature dependent rate constant of any chemical reaction as per Arrhenius equation is expressed as,

$$
K(D, T)=K_{0}(D) \exp \left(\frac{-E_{a}}{R T}\right)
$$

Where, $K$ is a rate constant of the reaction, $K_{0}$ is the pre-exponential factor, $E_{a}$ is activation energy (here considered as catalytic activation energy), $R$ is a universal gas constant and $T$ is the temperature.

At the melting temperature $\left(T_{m}\right)$, if rate constant $(K)$ is independent of melting temperature and size of the particle, the rate constant will be the same for all the nanoparticles of a given system. Therefore,

$$
K\left[\left(D, T_{m}(D)\right]=K\left[\infty, T_{m}(\infty)\right]\right.
$$

Where, $\mathrm{T}_{\mathrm{m}}(\mathrm{D})$ and $\mathrm{T}_{\mathrm{m}}(\infty)$ are the melting temperatures for size dependent (i.e., nanostructure) and bulk system, respectively.

By rewriting Eqns. 1 and 2, the following expression can be obtained, 
$K_{0}(D) \exp \left(\frac{-E_{a}(D)}{R T_{m}(D)}\right)=K_{0}(\infty) \exp \left(\frac{-E_{a}(\infty)}{R T_{m}(\infty)}\right)$

The effect of pre-exponential factor $K_{0}(D)$ on $K(D, T)$ is negligible in comparison to the $\exp \left[-E_{a}(D) /(R T)\right]$ hence using first order approximation, we can assume $K_{0}(D) \approx\left[K_{0}(\infty)\right]$. This leads to the expression,

$$
\frac{E_{a}(D)}{E_{a}(\infty)}=\frac{T_{m}(D)}{T_{m}(\infty)}
$$

The liquid-drop model and (BOLS) mechanism suggest that both melting temperature $T_{m}$ and cohesive energy $E_{C}$, are related to the bond energy of crystalline atoms hence $T_{m}(\infty) \alpha E_{C}(\infty)$ (Nanda et al., 2002, Sun, 2007). If the nature of the chemical bonds remains the same, this relationship can be written for nano scaled region i.e. $\frac{T_{m}(D)}{T_{m}(\infty)} \approx \frac{E_{C}(D)}{E_{C}(\infty)}$. Lu and Meng, (2010) modified the thermodynamic model proposed by Yang et al., (2008) and include the shape factor $(\lambda)$ to calculate size and shape dependent catalytic activation energy. From that consideration, one can get $\mathrm{E}_{\mathrm{C}}$ as a function of size and shape,

$$
\frac{E_{C}(D, \lambda)}{E_{C}(\infty)}=\left(1-\frac{1}{12 D / D_{0}-1}\right) \exp \left(-\frac{2 \lambda S_{b}}{3 R} \frac{1}{12 D / D_{0}-1}\right)
$$

Here, $S_{b}=E_{b} / T_{b}$ is the bulk coherent entropy of crystals with $\mathrm{T}_{\mathrm{b}}$ being the bulk solid-vapor transition temperature and $\mathrm{R}$ is the ideal gas constant. Due to the instability in semiconductor compounds, it is difficult to find the value of $S_{b}$ value therefore we have used the value of $S_{b}$ equal to the $13 R$ in our calculations (Yang and Jiang, 2006). The shape factor, $\lambda$ depends on the ratio of surface atoms to total atoms. For the different shapes, the value of the shape factor $(\lambda)$ is used from reference (Lu and Meng,
2010). In addition to the shape effect, the dimension dependence of $E_{C}$ for the different size of $\mathrm{TiO}_{2}, \mathrm{CeO}_{2}$ and $\mathrm{SnO}_{2}$ nanoparticles is also reported. In eqn. $5, D$ denotes the diameter of nanoparticles while $D_{0}$ is the diameter at which all atoms are located on surface. $D_{0}$ can be expressed as,

$D_{0}=2(3-d) h$

Where $h$ and $d$ denote the value of atomic diameter and different dimensions of nanoparticles, i.e. $d=0$ for spherical nanoparticles, $d=1$ for nanowires and $d=2$ for thin films (Li et al., 2004).

\section{Results and Discussion}

Figure 1[a] presents the variation of $E_{C}(D) / E_{C}(\infty)$ (ratio of size dependent activation energy to its bulk counterpart) of rutile $\mathrm{TiO}_{2}$ nanoparticles of different dimensions which shows that the catalytic activation energy decreases as the size of the $\mathrm{TiO}_{2}$ nanoparticle decreases. The value of activation energy rapidly decreases below 5 $\mathrm{nm}$. It is clear from this figure that after 15 $\mathrm{nm}$, the ratio of catalytic energies becomes almost constant. The figure also depicts that the catalytic activation energy is minimum for spherical nanoparticles while it is maximum for the two dimensional case. This indicates that the spherical nanoparticles can act as better catalysts compared to one and two dimensional nanostructures. The decrease in the catalytic activation energy with the decreasing size reveals efficiency of nanoparticles to catalyze any chemical reaction. Fig. $1[\mathrm{~b}]$ and Fig. $1[\mathrm{c}]$ present size and dimension dependence of $\mathrm{E}_{\mathrm{C}}(\mathrm{D}) / \mathrm{E}_{\mathrm{C}}(\infty)$ for another nanoparticles of this group $\mathrm{CeO}_{2}$ and $\mathrm{SnO}_{2}$ respectively. Both figures show similar behavior as observed in the case of $\mathrm{TiO}_{2}$ nanoparticles. However, it saturates after $10 \mathrm{~nm}$ in the case of $\mathrm{CeO}_{2}$ and $\mathrm{SnO}_{2}$ nanostructures in contrast to $\mathrm{TiO}_{2}$ nanostructure. Spherical nanoparticles indicate good catalytic performance rather than nanowires and thin films. 


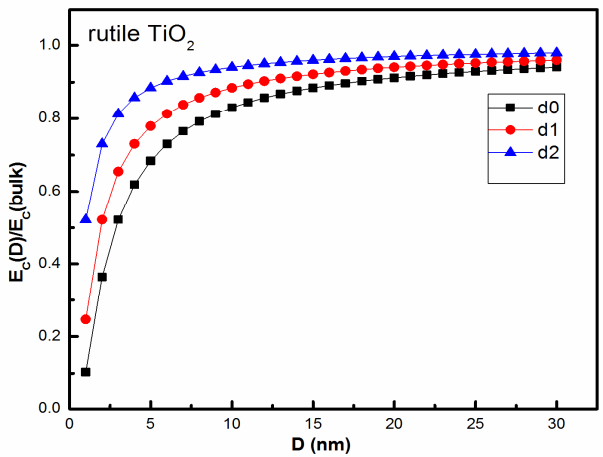

[a]

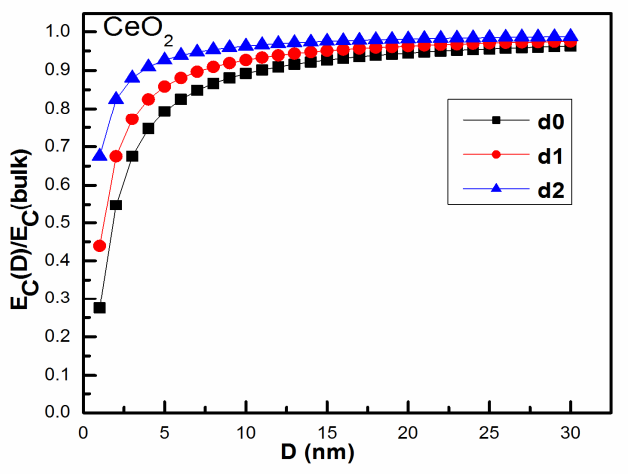

[b]

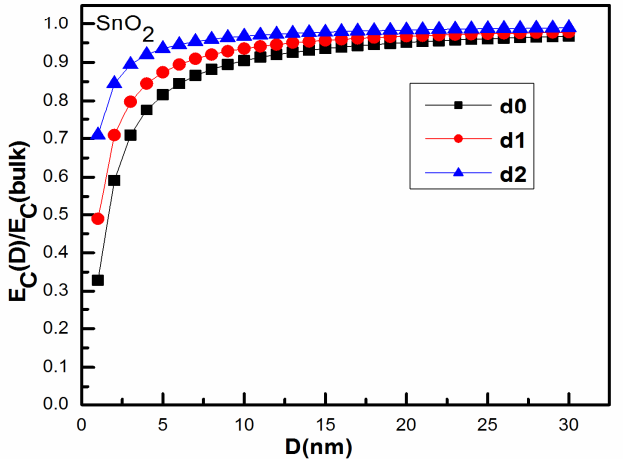

[c]

Figure 1: [a] $\mathrm{E}_{\mathrm{C}}(\mathrm{D}) / \mathrm{E}_{\mathrm{C}}(\infty)$ as a function of size and dimensions for rutile $\mathrm{TiO}_{2}$ using Eq.5. The related parameters in Eq.5 are $\mathrm{h}=0.3768 \mathrm{~nm}$ (Li et al., 2004), $\mathrm{S}_{\mathrm{b}}=13 \mathrm{R}=108.03 \mathrm{Jmol}^{-1} \mathrm{~K}^{-1}$ (Yang and Jiang, 2006). [b] $\mathrm{E}_{\mathrm{C}}(\mathrm{D}) / \mathrm{E}_{\mathrm{C}}(\infty)$ as a function of size and dimensions for $\mathrm{CeO}_{2}$ using Eq.5. The related parameters in Eq.5 are $\mathrm{h}=0.2342 \mathrm{~nm}$ (Yang and Li, 2008), $\mathrm{S}_{\mathrm{b}}=13 \mathrm{R}=108.03 \mathrm{Jmol}^{-1} \mathrm{~K}^{-1}$ (Yang and Jiang, 2006). [c] $\mathrm{E}_{\mathrm{C}}(\mathrm{D}) / \mathrm{E}_{\mathrm{C}}(\infty)$ as a function of size and dimensions for $\mathrm{SnO}_{2}$ using Eq.5. The related parameters in Eq.5 are $\mathrm{h}=0.2057 \mathrm{~nm}$ (Yang and Li, 2008), $\mathrm{S}_{\mathrm{b}}=13 \mathrm{R}=108.03 \mathrm{Jmol}^{-1} \mathrm{~K}^{-1}$ (Yang and Jiang, 2006).

Figure 2 shows the variation of $\mathrm{E}_{\mathrm{C}}(\mathrm{D}) / \mathrm{E}_{\mathrm{C}}(\infty)$ with size and different shapes of $\mathrm{TiO}_{2}$ nanoparticles. For a particular size, a tetrahedral shaped particle with (111) facet exhibits maximum value of $\mathrm{E}_{\mathrm{C}}$ compared to octahedral and cubic shaped nanoparticles. It can be observed from the figure that the cubic nanoparticles are having the minimum value of $E_{C}$ for a given size. This nature of catalytic activation energy indicates that the tetrahedral shaped nanoparticles are the most catalytically active. For $\mathrm{CeO}_{2}$ and $\mathrm{SnO}_{2}$ nanoparticles, we obtain similar behavior where tetrahedral shape of the particles show higher value of $E_{C}$ (not shown here). It can be explained by a fact that the small tetrahedral particles have sharp edges and corners (Narayanan and El-Sayed, 2004). It is found that the atoms located on these sites are chemically very active. In cubic nanoparticles, most of the atoms are located on (100) facets and found to be least active, consequently these particles possess higher value of $E_{C}$ (Narayanan and El-Sayed, 2004). 


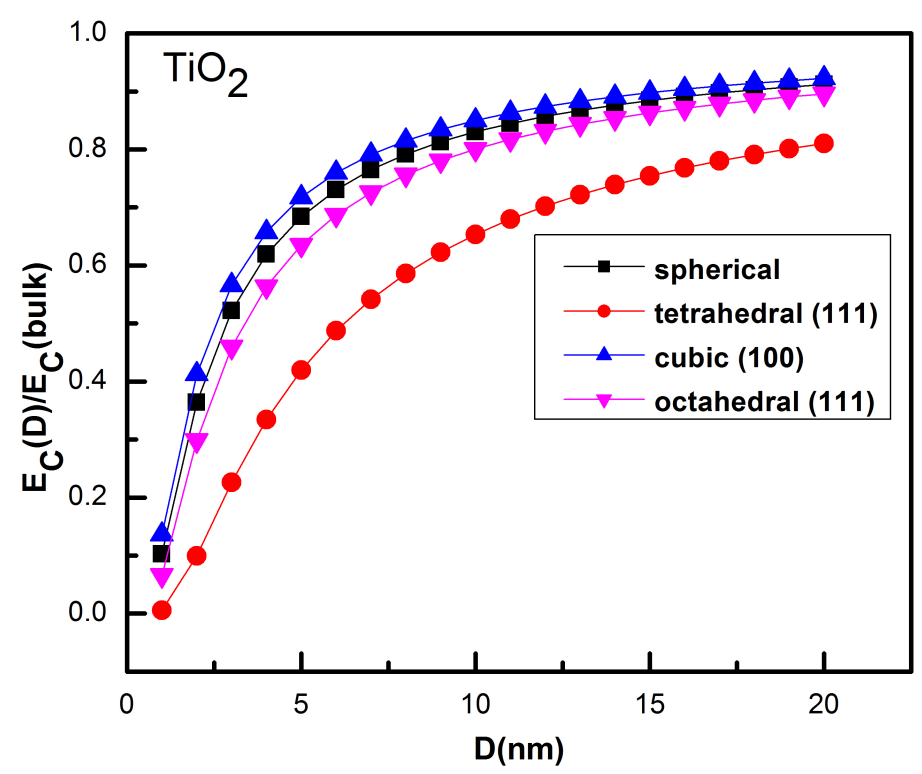

Figure 2: $\mathrm{E}_{C}(\mathrm{D}) / \mathrm{E}_{\mathrm{C}}(\infty)$ as a function of size and shapes for rutile $\mathrm{TiO}_{2}$ using $\mathrm{Eq}$. The related parameters in Eq. are $h=0.3768 \mathrm{~nm}$ (Li et al., 2004),$S_{b}=13 \mathrm{R}=108.03 \mathrm{Jmol}^{-1} \mathrm{~K}^{-1}$ (Yang and Jiang, 2006).

Figure 3 shows comparison of all the three tetrahedral shaped nanostructures (i.e., $\mathrm{TiO}_{2}$, $\mathrm{CeO}_{2}$ and $\mathrm{SnO}_{2}$ ) in terms of size dependent catalytic activation energy. It is clear from the figure that the $\mathrm{TiO}_{2}$ nanoparticles have minimum catalytic activation energy which implies that the nano structured $\mathrm{TiO}_{2}$ can be considered as an efficient candidate for catalytic activities. Although nano sized $\mathrm{SnO}_{2}$ has advantage for a particular reaction when used as a catalyst, it shows the least chemical activity in present study while $\mathrm{CeO}_{2}$ has the intermediate value of $\mathrm{E}_{\mathrm{C}}$ between $\mathrm{TiO}_{2}$ and $\mathrm{SnO}_{2}$. The similar trend of size and shape dependent catalytic activation energy is reported in case of platinum nanoparticles (Lu and Meng, 2010). 


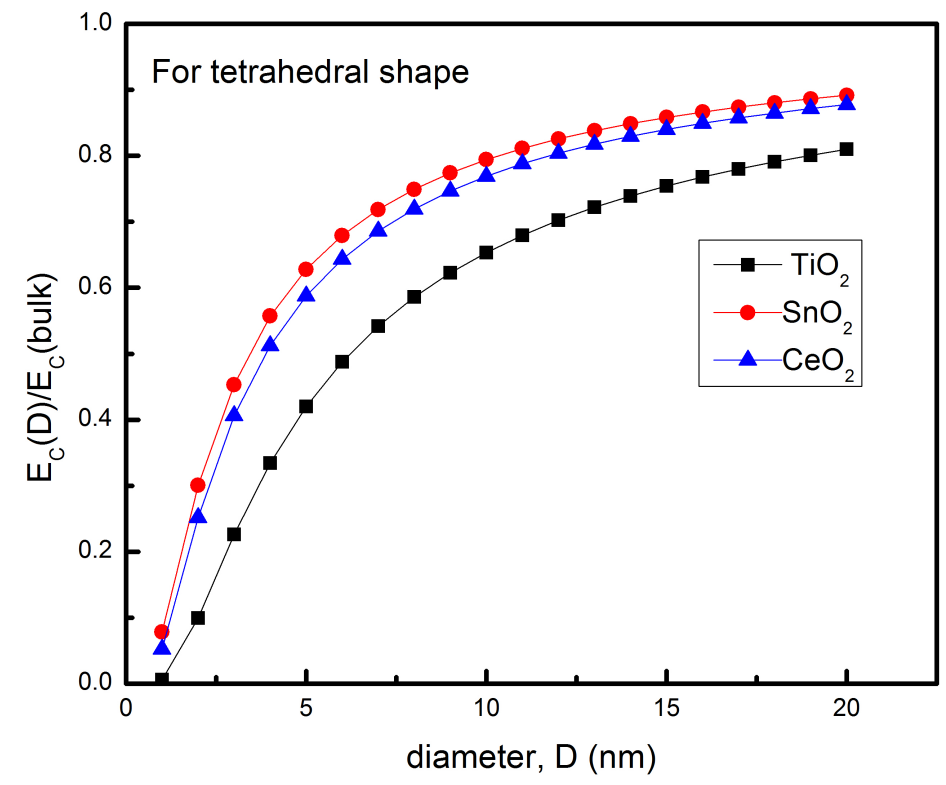

Figure 3: $\mathrm{E}_{\mathrm{C}}(\mathrm{D}) / \mathrm{E}_{\mathrm{C}}(\infty)$ as a function of size for tetrahedral shaped $\mathrm{TiO}_{2}, \mathrm{CeO}_{2}$ and $\mathrm{SnO}_{2}$ nanoparticles.

\section{Conclusion}

In summary, we have calculated the shape and size dependent catalytic activation energy of $\mathrm{TiO}_{2}, \quad \mathrm{SnO}_{2}$ and $\mathrm{CeO}_{2}$ nanostructures. The catalytic activation energy decreases with the decrease in particles' size. It becomes almost size independent when size of the particle exceeds 10 to $15 \mathrm{~nm}$. We observe that zero dimensional (i.e., spherical) nanoparticles have minimum catalytic activation energy than the two and one dimensional structures. The considerable difference is found for $E_{C}$ in the case of different shapes of nanoparticles, and tetrahedral shaped particles showed maximum efficiency for catalysis. In the case of $\mathrm{TiO}_{2}, \mathrm{CeO}_{2}$ and $\mathrm{SnO}_{2}$, $\mathrm{TiO}_{2}$ has minimum value of catalytic activation energy hence considered as better catalyst. Our study will find its implications in catalytic activity based applications.

\section{Acknowledgements}

One of the authors (PAB) acknowledges University Grants Commission (UGC), New Delhi for providing the financial assistance under Research Fellowship in Science for Meritorious Students (RFSMS) scheme.

\section{References}

1. Alivisatos, A.P. (1996) "Perspectives on the physical chemistry of semiconductor nanocrystals," The Journal of Physical Chemistry, 100 (31) 13226-13239.

2. Alivisatos, A.P, Barbara, P.F, Castleman, A.W, Chang, J, Dixon, D.A, Klein, M.L, McLendon, G.L, Miller, J.S, Ratner, M.A, Rossky, P.J, Stupp, S.I. and Thompson, M.E. (1998) "From molecules to materials: current trends and future directions," Advanced Materials, 10 (16) 1297-1336.

3. Astruc, D, Lu, F and Aranzaes, J.R. (2005) "Nanoparticles as recyclable catalysts: The frontier between homogeneous and 
heterogeneous catalysis ," Angewandte Chemie International Edition, 44 (48) 78527872.

4. Bell, A.T. (2003) "The impact of nanoscience on heterogeneous catalysis," Science, 299 (5613) 1688-1691.

5. Bhatt, P.A, Pratap, A. and Jha, P.K. (2012) "Study of size-dependent glass transition and Kauzmann temperatures of tin dioxide nanoparticles," Journal of Thermal Analysis and Calorimetry, 110 (2) 535-538.

6. Burda, C, Chen, X.B, Narayanan, R. and ElSayed, M.A. (2005) "Chemistry and properties of nanocrystals of different shapes," Chemical Reviews, 105 (4) 10251102.

7. Carrettin, S, Concepcion, P, Corma, A, Lopez Nieto, J.M. and Puntes, V.F. (2004) "Nanocrystalline $\mathrm{CeO}_{2}$ increases the activity of $\mathrm{Au}$ for $\mathrm{CO}$ Oxidation by two orders of magnitude," Angewandte Chemie International Edition, 43 (19) 2538-2540.

8. Chen, M. and Goodman, D.W. (2006) "Catalytically active gold: From nanoparticles to ultrathin films," Accounts of chemical research, 39 (10) 739-746.

9. Crooks, R.M, Zhao, M, Sun, L, Chechik, V. and Yeung, L.K. (2001) "Dendrimerencapsulated metal nanoparticles: synthesis, characterization, and applications to catalysis," Accounts of chemical research, 34 (3) 181-190.

10. Desai, R, Gupta, S.K, Mishra, S, Jha, P.K. and Pratap, A. (2011) "The synthesis of $\mathrm{TiO}_{2}$ nanoparticles by wet-chemical method and their photoluminescence, thermal and vibrational characterizations: effect of growth condition," International Journal of Nanoscience, 10 (6) 1249-1256.

11. Ertl, G, Knozinger, H. and Weitkamp, J. (ed.) (1997) Handbook of heterogeneous catalysis, Weinheim: Wiley-VCH.

12. Gleiter, H. (2000) "Nanostructured materials: basic concepts and microstructure," Acta Materialia, 48 (1) 1-29.
13. Guo, M.N, Guo, C.X, Jin, L.Y, Wang, Y.J, Li, J.Q. and Luo, M.F. (2010) "Nano-sized $\mathrm{CeO}_{2}$ with extra-high surface area and its activity for CO oxidation," Materials Letters, 64 (14) $1638-1640$

14. Gupta, S.K, Desai, R, Jha, P.K, Sahoo, S. and Kirin, D. (2010) "Titanium dioxide synthesized using titanium chloride: size effect study using Raman spectroscopy and photoluminescence," Journal of Raman Spectroscopy, 41 (3) 350-355.

15. Gupta, S.K. and Jha, P.K. (2009) "Modified phonon confinement model for size dependent Raman shift and linewidth of silicon nanocrystals," Solid State Communications, 149 (45-46) 1989-1992.

16. Gupta, S.K, Jha, P.K. and Arora, A.K. (2008) "Size dependent acoustic phonon dynamics of $\mathrm{Cd}$ Te 0.68 Se 0.32 nanoparticles in borosilicate glass," Journal of Applied Physics, 103 (124307) 1-6.

17. Gupta, S.K, Sahoo, S, Jha, P.K, Arora, A.K. and Azhniuk, Y.M. (2009) "Observation of torsional mode in $\mathrm{CdS}_{1-\mathrm{x}} \mathrm{Se}_{\mathrm{x}}$ nanoparticles in a borosilicate glass," Journal of Applied Physics, 106 (024307) 1-7.

18. Gupta, S.K, Talati, M. and Jha, P.K. (2008) "Shape and size dependent melting point temperature of nanoparticles," Materials Science Forum, 570, 132-137.

19. Jha, P.K. (ed.) (2013) Titanium Dioxide: Applications, synthesis and toxicity, New York: Nova Science Publications.

20. Jha, P.K. and Talati, M. (2006) "Phonons in nanocrystal quantum dots," in Chang, J.V. (ed.) Focus on Condensed Matter Physics Research, New York:Nova Science Publishers.

21. Jiang, Q. and Lu, H.M. (2008) "Size dependent interface energy and its applications," Surface Science Reports, 63 (10) 427-464.

22. Jiang, Q Zhang, S.H. and Li, J.C. (2004) "Grain size-dependent diffusion activation 
energy in nanomaterials," Solid State Communications, 130 (9) 581-584.

23. Kung, M.C, Davis, R.J. and Kung, H.H. (2007) "Understanding Au-catalyzed lowtemperature CO oxidation," The Journal of Physical Chemistry C, 111 (32) 11767-11775.

24. Lewis, L.N. (1993) "Chemical catalysis by colloids and clusters," Chemical Reviews, 93 (8) 2693-2730.

25. Li, G, Boerio-Goates, J, Woodfield, B.F. and Li, L. (2004) "Evidence of linear lattice expansion and covalency enhancement in rutile TiO2 nanocrystals," Applied Physics Letters, 85 (11) 2059-2061.

26. Li, Y, Petroski, J. and El-Sayed, M.A (2000) "Activation energy of the reaction between hexacyanoferrate(III) and thiosulfate ions catalyzed by platinum nanoparticles," The Journal of Physical Chemistry B, 104 (47) 10956-10959.

27. Lu, H.M. and Meng, X.K. (2010) "Theoretical model to calculate catalytic activation energies of platinum nanoparticles of different sizes and shapes," The Journal of Physical Chemistry C, 114 (3) 1534-1538.

28. Mankad, V, Gupta, S.K. and Jha, P.K. (2011) "Low frequency raman scattering of anatase titanium dioxide nanocrystals," Physica E, 44 (3) 614-617.

29. Mishra, S, Jha, P.K. and Pratap, A. (2012) "Study of size-dependent glass transition and Kauzmann temperature of titanium dioxide nanoparticles," Journal of Thermal Analysis and Calorimetry, 107 (1) 65-68.

30. Nanda, K.K, Sahu, S.N. and Behera, S.N. (2002) "Liquid-drop model for the sizedependent melting of low-dimensional systems," Physical Review A, 66 (013208) 18.

31. Narayanan, R. and El-Sayed, M.A. (2004) "Shape-dependent catalytic activity of platinum nanoparticles in colloidal solution," Nano Letters, 4 (7) 1343-1348.
32. Roucoux, A, Schulz, J. and Patin, H. (2002) "Reduced transition metal colloids: A novel family of reusable catalysts?," Chemical reviews, 102 (10) 3757-3778.

33. Schmid, G. (ed.) (2005) Nanoparticles: From theory to application, Weinheim: Wiley-VCH.

34. Sharghi, H, Ebrahimpourmoghaddam, S, Memarzadeh, R. and Javadpour, S. (2013) "Tin oxide nanoparticles (NP-SnO $)_{2}$ : preparation, characterization and their catalytic application in the Knoevenagel condensation," Journal of the iranian chemical society, 10 (1) 141-149.

35. Somorjai, G.A, Contreras, A.M, Montano, M. and Rioux, R.M. (2006) "Clusters, surfaces, and catalysis," Proceedings of The National Academy of Sciences of the United States of America, 103 (28) 10577-10583.

36. Sun, C.Q. (2007) "Size dependence of nanostructures: Impact of bond order deficiency," Progress in Solid State Chemistry, 35 (1) 1-159.

37. Talati, M. and Jha, P.K. (2005) "Lowfrequency acoustic phonons in nanometric $\mathrm{CeO}_{2}$ particles," Physica E, 28 (2) 171-177.

38. Wasylenko, W. and Frei, H. (2007) “ Direct observation of the kinetically relevant site of CO hydrogenation on supported Ru catalyst at $700 \mathrm{~K}$ by time-resolved FT-IR spectroscopy," Physical Chemistry Chemical Physics, 9 (40) 5497-5502.

39. Yang, C.C, Armellin, J. and Li, S. (2008) "Determinants of thermal conductivity and diffusivity in nanostructural semiconductors," The Journal of Physical Chemistry B, 112 (5) 1482-1486.

40. Yang, C.C. and Jiang, Q. (2006) "Size effect on the bandgap of II-VI semiconductor nanocrystals," Materials Science and Engineering B, 131 (1-3) 191-194.

41. Yang, C.C. and Li, S. (2008) "Sizedependent raman red shifts of 
semiconductor nanocrystals," The Journal of Physical Chemistry B, 112 (45) 1419314197.

42. Zhang, Z, Li, J.C. and Jiang, Q. (2000) "Modeling for size-dependent and dimension-dependent melting of nanocrystals," Journal of Physics D: Applied Physics, 33 (20) 2653-2656.
43. Zhou, X, Xu, W, Liu, G, Panda, D. and Chen, P. (2010) "Size-dependent catalytic activity and dynamics of gold nanoparticles at the single-molecule level," Journal of the American Chemical Society, 132 (1) 138-146. 